\title{
Sine-Gordon ratchets with general periodic, additive, and parametric driving forces
}

\author{
Elías Zamora-Sillero, ${ }^{1, *}$ Niurka R. Quintero, ${ }^{1, \dagger}$ and Franz G. Mertens ${ }^{2, \$}$ \\ ${ }^{1}$ Departamento de Física Aplicada I, E. U. P., Universidad de Sevilla, Virgen de África 7, 41011 Sevilla, Spain \\ ${ }^{2}$ Physikalisches Institut, Universität Bayreuth, D-85440, Germany
}

(Received 3 July 2007; published 6 December 2007)

\begin{abstract}
We study the soliton ratchets in the damped sine-Gordon equation with periodic nonsinusoidal, additive, and parametric driving forces. By means of symmetry analysis of this system we show that the net motion of the kink is not possible if the frequencies of both forces satisfy a certain relationship. Using a collective coordinate theory with two degrees of freedom, we show that the ratchet motion of kinks appears as a consequence of a resonance between the oscillations of the momentum and the width of the kink. We show that the equations of motion that fulfill these collective coordinates follow from the corresponding symmetry properties of the original systems. As a further application of the collective coordinate technique we obtain another relationship between the frequencies of the parametric and additive drivers that suppresses the ratchetlike motion of the kink. We check all these results by means of numerical simulations of the original system and the numerical solutions of the collective coordinate equations.
\end{abstract}

DOI: 10.1103/PhysRevE.76.066601

PACS number(s): 05.45.Yv, 05.60.Cd, 63.20.Pw

\section{INTRODUCTION}

Transport phenomena of ratchet type in nonlinear systems are a topic of interest in many fields of physics. Although this phenomenon has been proposed first to explain the unidirectional motion of molecular motors taking place inside the cells [1], nowadays the net motion of fluxons in long Josephson junctions (JJs) [2-6] or vortices in superconductors [7], the motion of cold atoms in optical lattices [8], the rectification of Brownian motion [9,10], just to name a few examples, can be analyzed within the framework of this phenomenon.

The motion of particles or solitons when the average of external forces is zero appears, in general, as a consequence of the breaking of certain symmetries in each particular system, which should be at nonequilibrium [11,12]. The asymmetry can be induced by a ratchet potential, whereas an ac force is used simultaneously in order to get out of the equilibrium of the system [13-15]. On the other hand, using a biharmonic force that takes the system out of equilibrium and at the same time breaks certain temporal symmetries, instead of spatial ones, seems to be more appropriate from the experimental point of view $[5,8,16]$. Both kinds of ratchet systems have been extensively studied in the case of particles or solitons using symmetry analysis $[13,17]$, collective coordinates in the case of extended models [18-21], numerical simulations, and also experimental realizations [5,6,8,22-24]. More complex ratchet systems appear when the asymmetry is introduced using both a ratchet potential and a biharmonic force $[25,26]$.

In a recent paper [27] using a collective coordinate technique we have studied the ratchet motion of a kink in a damped sine-Gordon (SG) system, driven by two ac (sinusoidal) forces, both single harmonics, one was added as a

\footnotetext{
*ezamora80@us.es

†niurka@us.es

franz.mertens@uni-bayreuth.de
}

parametric perturbation and the other one in an additive way. The results from the symmetry analysis carried out in $[18,28]$ cannot be applied to this system because of the parametric periodic force. The goal of the present work is to provide a symmetry analysis in order to predict the appearance of the ratchet phenomenon in this kind of system and also to generalize the previous studies on the sinusoidal driving forces [18-20,27-29] to the case of nonsinusoidal ones.

With this aim in mind we study the ratchet effect in a damped sine-Gordon system with generic additive and parametric periodic driving forces, where the analysis will not be restricted to the case of sinusoidal driving forces. In this regard recent studies using most general periodic functions (including elliptic functions) $[25,30]$ have been done with the aim to optimize the transport in ratchet devices.

The damped sine-Gordon system with generic additive and parametric periodic driving forces is represented by the following equation:

$$
\phi_{t t}-\phi_{x x}+\frac{d U}{d \phi}\left[1+\epsilon_{1} g(t)\right]=-\beta \phi_{t}+\epsilon_{2} f(t),
$$

where the subindices denote the partial derivatives of the field with respect to space $x$ and time $t, U(\phi)=1-\cos (\phi)$ is the sine-Gordon potential, $\epsilon_{1}$ and $\epsilon_{2}$ are the amplitudes of the parametric and additive $T_{1^{-}}$and $T_{2}$-periodic forces, $g(t)$ and $f(t)$ respectively; and $\beta$ is a damping coefficient. To fully specify the problem, this equation must be amended by initial conditions for $\phi(x, 0)$ and $\phi_{t}(x, 0)$ and boundary conditions. In particular we use the aperiodic boundary conditions given by

$$
\begin{gathered}
\lim _{x \rightarrow+\infty} \phi(x, t)=\lim _{x \rightarrow-\infty} \phi(x, t)+Q, \\
\lim _{x \rightarrow+\infty} \phi_{x}(x, t)=\lim _{x \rightarrow-\infty} \phi_{x}(x, t),
\end{gathered}
$$

where $Q=2 \pi$ is the so-called topological charge. Denoting $A(t)=\lim _{x \rightarrow-\infty} \phi(x, t)$ one can check that this function satisfies 


$$
A_{t t}+\frac{d U}{d \phi}(A)\left[1+\epsilon_{1} g(t)\right]=-\beta A_{t}+\epsilon_{2} f(t) .
$$

Note that the invariance of our system under certain symmetry transformations requires not only that Eq. (1) does not change under the symmetry transformations but also that Eqs. (2) and (3) remain invariant [31]. This model is also of interest because we can recover from it the soliton ratchets induced either by a biharmonic force $[18,20,28]$ or by parametric and additive ac forces $[19,27,29]$. Once the working model is defined, in the next section a symmetry analysis is provided to obtain the necessary conditions that must be fulfilled by the two periodic forces to get a net motion of the kink. Further on in Sec. III a collective coordinate (CC) theory with two collective variables, namely the position $X(t)$ and the width $l(t)$ of the kink, is used with the aim, first, of obtaining the mechanism underlying this kind of soliton ratchets and, second, of providing a symmetry analysis for the collective coordinate equations. In the next section some further applications related to this technique are presented. In Sec. V we verify our analytical results by means of direct numerical simulations. Finally, to conclude the paper in Sec. VI we summarize the main results of the previous sections.

\section{SYMMETRY CONDITIONS FOR ZERO VELOCITY}

In this section, by using a symmetry analysis of our working model, we obtain the necessary conditions that must be fulfilled by the forces $g(t)$ and $f(t)$ to get a net motion of the kink.

Generalizing the symmetry transformations proposed in $[18,28]$ for a damped SG equation driven only by a biharmonic force, it is easy to check that the following symmetry transformations,

$$
\begin{gathered}
x \rightarrow-x, \\
t \rightarrow t+m T_{1}, \\
\phi \rightarrow-\phi+Q,
\end{gathered}
$$

leave Eqs. (1)-(3) unchanged and change the sign of the kink velocity

$$
V=\frac{1}{Q} \int_{-\infty}^{+\infty} d x x \phi_{x t}
$$

only if

$$
\begin{gathered}
U(\phi-Q / 2)=U(-\phi+Q / 2), \\
f\left(t+m T_{1}\right)=-f(t) .
\end{gathered}
$$

Notice that Eq. (6) is always fulfilled for SG potential. If we assume that the $T_{2}$-periodic force $f(t)$ also satisfies

$$
f\left(t+T_{2} / 2\right)=-f(t),
$$

as has been considered in $[19,27,29]$, the condition (7) is equivalent to

$$
\frac{\omega_{1}}{\omega_{2}}=\frac{2 m}{2 n+1}, \quad m=1,2,3, \ldots ; \quad n=0,1,2, \ldots .
$$

Hence if the frequencies of $g(t)$ and $f(t), \omega_{1}$ and $\omega_{2}$, respectively, satisfy Eq. (9), the time-averaged velocity defined as

$$
\langle V\rangle=\lim _{\tau \rightarrow+\infty} \frac{1}{\tau} \int_{0}^{\tau} d t V(t),
$$

is expected to be zero. Or, if $f(t)$ does not fulfill the condition (8) and/or the relation (9) between the two frequencies $\omega_{1}$ and $\omega_{2}$ does not hold, a net motion of the kink could appear.

\section{SYMMETRY ANALYSIS OF THE CC EQUATIONS}

The symmetry approach carried on in the previous section, although useful for predicting the unidirectional motion of the kink in the system modeled by Eqs. (1)-(3), does not provide information about the mechanisms underlying the soliton ratchets. In many problems of soliton ratchets type the CC technique has allowed not only to find out why the soliton moves under an external force with zero time average, but also provides an approximate expression for the average velocity. To this end, in Refs. $[18,19,32]$ an ansatz for an approximate solution was used, which depends on the center of the kink and its width. Thus using collective coordinate theories $[33,34]$ it has been possible to obtain the equations of motions that satisfy these variables.

In order to solve Eq. (1) we can use the Rice ansatz [32]

$$
\phi(x, t)=4 \arctan \left(\exp \left[\frac{x-X(t)}{l(t)}\right]\right),
$$

as the approximate kink solution of the perturbed SG equation; where $X(t)$ represents the center of the kink and $l(t)$ its width. Following, for instance, the Lagrangian formalism developed in [34] we obtain the oridinary differential equations (ODEs)

$$
\begin{gathered}
\frac{M_{0} l_{0}}{l(t)} \ddot{X}-\frac{M_{0} l_{0}}{l(t)^{2}} \dot{X} \dot{l}=-\beta \frac{M_{0} l_{0}}{l(t)} \dot{X}-Q \epsilon b f(t), \\
\alpha\left[\dot{l}^{2}-2 \beta l i-2 \ddot{l}\right]-\frac{l^{2}}{l_{0}^{2}}\left(\frac{l_{0}^{2}}{l^{2}} \dot{X}^{2}+1\right)+1-\frac{l^{2}}{l_{0}^{2}} \epsilon a g(t)=0,
\end{gathered}
$$

where we have introduced the factors $a \equiv \epsilon_{1} / \epsilon$ and $b \equiv \epsilon_{2} / \epsilon$, $\epsilon$ being a rescaling parameter. For SG $\alpha=\pi^{2} / 12, M_{0}=8$, and $l_{0}=1$ are the mass and the width of the kink at rest, respectively. It should be stressed that Eqs. (12) and (13) also hold for the $\phi^{4}$ system, where the potential $U(\phi)=(1 / 4)\left(1-\phi^{2}\right)^{2}$, the Rice ansatz reads $\phi(x, t)=\tanh \{[x-X(t)] l(t)\}$ and the corresponding parameters are given by $\alpha=\left(\pi^{2}-6\right) / 12, Q=2$, $M_{0}=4 /(3 \sqrt{2})$, and $l_{0}=\sqrt{2}$, and so a similar analysis can be done for the $\phi^{4}$ model.

Recently we have studied the ratchet dynamics of the kink induced by a biharmonic force in the case of sine-Gordon, which does not have any internal mode $[35,36]$, and the net motion of the kink in the $\phi^{4}$ model [37], which has an inter- 
nal mode. In both systems we found out not only the same ratchet mechanism for the net motion of the kink, but also that the average velocity as a function of the biharmonic force and the damping coefficient exhibits the same qualitative behavior. Because of this we do not expect any new findings if we work with a $\phi^{4}$ potential instead of the sineGordon potential, and therefore, although this theory can be applied straightforwardly to the $\phi^{4}$ equation, from now on we will study in detail only the sine-Gordon model represented by Eq. (1).

Note that after the change of variable

$$
\frac{d X}{d t}=\frac{P(t) l(t)}{M_{0} l_{0}},
$$

where $P(t) \equiv-\int_{-\infty}^{+\infty} d x \phi_{x} \phi_{t}$ represents the momentum of the system, Eq. (12) becomes a linear equation for $P(t)$,

$$
\frac{d P}{d t}=-\beta P(t)-q \epsilon b f(t),
$$

which can be solved exactly, yielding after a transient time $t \gg 1 / \beta$,

$$
P(t)=-q \epsilon b \int_{0}^{t} d \tau \exp [\beta(\tau-t)] f(\tau) .
$$

If $f(t)$ satisfies the time-shift symmetries (7) and (8), after a transient time $t \gg 1 / \beta$, we note that the symmetry transformations proposed in Eq. (4) leave invariant Eq. (13), but simultaneously the solution $P$ changes its sign. Therefore if the frequencies of both drivers satisfy Eq. (9), the timeaveraged velocity

$$
\langle V\rangle=\lim _{\tau \rightarrow+\infty} \frac{1}{\tau} \int_{0}^{\tau} \frac{P(t) l(t)}{M_{0} l_{0}} d t,
$$

also changes its sign and so a net motion of the kink is not possible.

It is important to remark that even when the Rice ansatz (11) does not satisfy the boundary conditions (2) of Eq. (1) this theory has allowed us to establish that the nonlinear coupling between the momentum $P(t)$ of the kink and the internal oscillations $l(t)$ of the kink width is the main mechanism of this kind of soliton ratchet [see Eq. (17)] [20,27,37]. Moreover, from Eq. (12) it is clear that, although the additive periodic force $f(t)$ has zero average, the center of the kink feels an effective periodic force with a time average different from zero [37].

Since the symmetries of the system are a very important issue regarding the ratchet phenomenology, the fact that the $\mathrm{CC}$ theory succeeds in representing the corresponding symmetry properties of the original system is crucial for the correct description of the phenomena. In this regard, another important issue to remark is related with the fact that this collective coordinate theory is equivalent to the variation of the momentum and the energy of the system [38]. Further analysis of the CC equations of type (13)-(15) has been performed in [27], where a perturbation theory has been used in order to find $l(t)$ and finally to obtain an approximate expression for the average velocity.
To proceed we assume that, after the transient time, $l(t)$ oscillates around the unperturbed initial width $l_{0}$ of the static kink. Then this function is expanded in powers of $\epsilon$ around $l_{0}$,

$$
l(t)=l_{0}+\sum_{n=1}^{\infty} \epsilon^{n} l_{n}(t)
$$

Note that despite the sine-Gordon equation does not have any internal mode, the oscillations of the width of the kink are related to the excitation of certain phonon modes [36]. After that, the solution of Eq. (15) and Eqs. (14) and (18) are inserted in Eq. (13) and a hierarchy of equations in powers of $\epsilon$ is obtained. In order $O(\epsilon)$

$$
\mathcal{L} l_{1}=-\frac{\Omega_{R}^{2} a l_{0}}{2} g(t),
$$

and for the next-order corrections $(n \geq 1)$ straightforward calculations show that

$$
\begin{aligned}
\mathcal{L} l_{n+1}= & \frac{\sum_{m=1}^{n} i_{m} i_{n+1-m}}{2 l_{0}}-\frac{\beta}{l_{0}} \sum_{m=1}^{n} l_{m} i_{n+1-m}-\frac{\Omega_{R}^{2}}{2 l_{0}} \\
& \times\left[\sum_{m=1}^{n} l_{m} l_{n+1-m}+\frac{1}{M_{0}^{2}}\left(\sum_{m=0}^{n-1} l_{m} l_{n-1-m}\right) \widetilde{P}^{2}\right] \\
& -\frac{1}{l_{0}} \sum_{m=1}^{n} l_{m} \ddot{l}_{n+1-m}-\frac{a g(t) \Omega_{R}^{2}}{2 l_{0}}\left(\sum_{m=0}^{n} l_{m} l_{n-m}\right),
\end{aligned}
$$

where $\mathcal{L} \equiv d^{2} / d t^{2}+\beta d / d t+\Omega_{R}^{2}$ is a second-order linear differential operator, $\Omega_{R}=1 / \sqrt{\alpha} l_{0}$ is the so-called Rice frequency [32], and $\widetilde{P}=P / \epsilon$. It is easy to verify that after the symmetry transformations $t \rightarrow t+m T_{1}$, this hierarchy of equations remains unchanged for all $l_{n}$ if $f(t)$ fulfills the condition (7) and therefore the aforementioned symmetry properties are preserved after using this perturbation theory. Other interesting consequences of the CC technique are presented in the next section.

\section{FURTHER APPLICATIONS OF THE CC TECHNIQUE}

Coming back to the condition (9) for the suppression of any net motion, a remarkable question arises: Can any other relationship between these two frequencies suppress the unidirectional motion of the kink?

On the other hand, this condition predicts a zero-average velocity when $\omega_{2}=\omega_{1} / 2$, however, interchanging the two frequencies in Eq. (9) and choosing $\omega_{1}=\omega_{2} / 2$ a net motion of the kink can appear as has recently been shown in [27]. In this sense we can say that these two frequencies are not equivalent. For the following we define that the parametric and additive periodic forces are equivalent if it is possible to interchange them and to obtain in both cases the same approximate nonzero average velocity. The other question that we would like to address here is the following: Under which restrictions can the parametric and additive periodic forces be considered equivalent? 


\section{A. Zero average velocity under not commensurable frequencies}

If the additive $T_{2}$-periodic force also satisfies $f(t)=-f(t$ $+T_{2} / 2$ ) we show that it is necessary to break the condition (9) for the appearance of a net motion of the kink. This condition implies that the relation between the frequencies of the parametric and additive drivers is equal to a certain rational number. In this section we will show, by using the CC equations derived in the previous section, that the ratchet effect in Eq. (1) does not appear when the frequencies of the two periodic forces $g(t)$ and $f(t)\left[f\left(t+T_{2} / 2\right)=-f(t)\right]$ are related by

$$
\frac{\omega_{2}}{\omega_{1}}=s \in \mathbb{I} .
$$

Formally, the Fourier series of the $T_{1}$ - and $T_{2}$-periodic forces, $g(t)$ and $f(t)$, respectively, can be written as

$$
g(t)=\sum_{k=1}^{\infty} c_{k} \sin \left(k \omega_{1} t\right)+d_{k} \cos \left(k \omega_{1} t\right)
$$

and

$$
f(t)=\sum_{k=1}^{\infty} a_{k} \sin \left(k \omega_{2} t\right)+b_{k} \cos \left(k \omega_{2} t\right),
$$

where the coefficients $a_{2 k}=0$ and $b_{2 k}=0$ due to $f(t)$ $=-f\left(t+T_{2} / 2\right)$. The solution for $P(t)$ in Eq. (16) now reads

$$
\begin{aligned}
P(t)= & -b Q \epsilon \sum_{p=1}^{\sim} \frac{a_{p}}{\sqrt{\beta^{2}+\left(p \omega_{2}\right)^{2}}} \sin \left(p \omega_{2} t+\delta_{p}\right) \\
& +\frac{b_{p}}{\sqrt{\beta^{2}+\left(p \omega_{2}\right)^{2}}} \sin \left(p \omega_{2} t+\psi_{p}\right),
\end{aligned}
$$

where $\tilde{\Sigma}$ denotes the sum over odd numbers $p=2 k+1$ with $k=0,1,2, \ldots, \delta_{p}=-\arctan \left(\frac{\omega_{2} p}{\beta}\right), \psi_{p}=\arctan \left(\frac{\beta}{p \omega_{2}}\right)$. This implies that $P$ has harmonics with frequencies $(2 k+1) s \omega_{1}, k \in \mathbb{Z}$. At the same time, from Eqs. (19) and (20) it can be shown that the frequencies of any $l_{j}$ belong to the set $\left\{\left(r+2 m_{1} s\right) \omega_{1}\right\}$, where $r, m_{1} \in \mathbb{Z}$. Hence using the expression (17) for the average velocity, one realizes that a nonzero average velocity appears, if first the oscillatory functions $P(t)$ and $l(t)$ have at least one common frequency; but this implies that

$$
r=s\left[2\left(k-m_{1}\right)+1\right], \quad m_{1}, k \in \mathbb{Z},
$$

which is a contradiction because $r \in \mathbb{Z}$. Thus we have shown, within the framework of the $\mathrm{CC}$, that the time-averaged velocity, given by Eq. (17), is zero when the frequencies of $g(t)$ and $f(t)$ satisfy Eq. (21). Bear in mind that if $f(t) \neq-f(t$ $+T_{2} / 2$ ), at least one of the coefficients in the Fourier series, either $a_{2 k}$ or $b_{2 k}$, should be different from zero. This implies that $P(t)$ oscillates with frequencies $p \omega_{2}$, where $p \in \mathbb{N}(p$ $\neq 0)$, while $l_{2}$ oscillates with frequencies $2 p \omega_{2}$ due to the term $P^{2}$ in Eq. (20). Therefore since $P(t)$ and $l_{2}$ share common frequencies, they could resonate and induce a net transport of the kinks, even if $\omega_{1}$ and $\omega_{2}$ satisfy either the relation (9) or (21).

\section{B. Conditions of equivalence between additive and parametric forces}

With the aim of investigating whether the interchange of the two forces does not affect the ratchet velocity, we use the Fourier series of $g(t)$ and $f(t)$, given by Eqs. (22) and (23), respectively. Nevertheless, we will not restrict our study to the case where the additive periodic force $f(t)$ satisfies $f(t)$ $=-f\left(t+T_{2} / 2\right)$, so all the coefficients $a_{2 k}$ and $b_{2 k}$ in the Fourier series for $f(t)$ cannot vanish simultaneously.

The solution (16) for $P(t)$ now reads

$$
\begin{aligned}
P(t)= & -b \epsilon Q \sum_{p=1} \frac{a_{p}}{\sqrt{\beta^{2}+\left(p \omega_{2}\right)^{2}}} \sin \left(p \omega_{2} t+\delta_{p}\right) \\
& +\frac{b_{p}}{\sqrt{\beta^{2}+\left(p \omega_{2}\right)^{2}}} \sin \left(p \omega_{2} t+\psi_{p}\right),
\end{aligned}
$$

where $p$ is any integer in contrast to the expression (24) where $p$ is odd.

Substituting Eq. (22) in Eq. (19), it is straightforward to show that the first-order correction of the kink width reads

$$
\begin{aligned}
l_{1}= & -\frac{\Omega_{R}^{2} l_{0} a}{2} \sum_{k} \frac{c_{k}}{\sqrt{\left[\Omega_{R}^{2}-\left(k \omega_{1}\right)^{2}\right]^{2}+\left(k \omega_{1} \beta\right)^{2}}} \sin \left(k \omega_{1} t+\lambda_{k}\right) \\
& +\frac{d_{k}}{\sqrt{\left[\Omega_{R}^{2}-\left(k \omega_{1}\right)^{2}\right]^{2}+\left(k \omega_{1} \beta\right)^{2}}} \sin \left(k \omega_{1} t+\chi_{k}\right)
\end{aligned}
$$

where $\lambda_{k}=-\arctan \left(\frac{k \beta \omega_{1}}{\Omega_{R}^{2}-\left(k \omega_{1}\right)^{2}}\right)$ and $\chi_{p}=\arctan \left(\frac{\Omega_{R}^{2}-\left(k \omega_{1}\right)^{2}}{k \beta \omega_{1}}\right)$. Substituting Eqs. (26) and (27) in Eq. (17), after some straightforward calculations the average velocity reads

$$
\begin{aligned}
\langle V\rangle= & \frac{\epsilon^{2} a b Q \Omega_{R}^{2}}{4 M_{0}} \\
& \times \sum_{k} \frac{1}{\sqrt{\left[\beta^{2}+\left(k \omega_{1}\right)^{2}\right]\left\{\left[\Omega_{R}^{2}-\left(k \omega_{1}\right)^{2}\right]^{2}+\left(\beta k \omega_{1}\right)^{2}\right\}}} \\
& \times\left\{a_{k q} c_{k} \cos \left(\delta_{k q}-\lambda_{k}\right)+a_{k q} d_{k} \cos \left(\delta_{k q}-\chi_{k}\right)\right. \\
& \left.+b_{k q} c_{k} \cos \left(\psi_{k q}-\lambda_{k}\right)+b_{k q} d_{k} \cos \left(\psi_{k q}-\chi_{k}\right)\right\}+O\left(\epsilon^{3}\right),
\end{aligned}
$$

where we have assumed that $\omega_{1}=q \omega_{2}$, being $q \in \mathbb{Q}$; the sum runs over $k$ such that $k q \in \mathbb{Z}, \delta_{k q}=-\arctan \left(\frac{k \omega_{1}}{\beta}\right)$, and $\psi_{k q}$ $=\arctan \left(\frac{\beta}{k \omega_{1}}\right)$. Note that, if $q=2 m /(2 n+1)(n=0,1,2, \ldots$ and $m=1,2, \ldots)$ the average velocity in order $O\left(\epsilon^{2}\right)$ vanishes as is expected from the symmetry analysis carried out in Sec. II.

Interchanging the parametric and additive forces, the collective variables $X(t)$ and $l(t)$ and the momentum $P(t)$ satisfy Eqs. (12), (13), and (15), respectively, with $f$ and $g$ interchanged. The equation for the first order of $l(t)$ reads as Eq. (19) exchanging $b$ for $a$ and $f$ for $g$. Therefore the solutions for $P(t)$ and $l_{1}(t)$ are represented by Eqs. (26) and (27), respectively, interchanging $a$ with $b$, and $\omega_{2}$ with $\omega_{1}$. Taking into account that $\omega_{1}=q \omega_{2}$, straightforward calculations show that the average velocity reads 


$$
\begin{aligned}
\langle V\rangle= & \frac{\epsilon^{2} a b Q \Omega_{R}^{2}}{4 M_{o}} \\
& \times \sum_{p} \frac{1}{\sqrt{\left[\beta^{2}+\left(p \omega_{1}\right)^{2}\right]\left\{\left[\Omega_{R}^{2}-\left(p \omega_{1}\right)^{2}\right]^{2}+\left(\beta p \omega_{1}\right)^{2}\right\}}} \\
& \times\left\{a_{p q} c_{p} \cos \left(\tilde{\delta}_{p}-\tilde{\lambda}_{p q}\right)+c_{p} b_{p q} \cos \left(\tilde{\delta}_{p}-\tilde{\chi}_{p q}\right)\right. \\
& \left.+a_{p n} d_{p} \cos \left(\widetilde{\psi}_{p}-\tilde{\lambda}_{p q}\right)+b_{p q} d_{p} \cos \left(\tilde{\psi}_{p}-\tilde{\chi}_{p q}\right)\right\}+O\left(\epsilon^{3}\right),
\end{aligned}
$$

where the sum runs over $p$ such that $p q \in \mathbb{Z}$ and

$$
\begin{gathered}
\tilde{\lambda}_{p q}=-\arctan \left(\frac{\beta p q \omega_{2}}{\Omega_{R}^{2}-\left(p q \omega_{2}\right)^{2}}\right) \\
=-\arctan \left(\frac{\beta p \omega_{1}}{\Omega_{R}^{2}-\left(p \omega_{1}\right)^{2}}\right)=\lambda_{p}, \\
\tilde{\chi}_{p q}=\arctan \left(\frac{\Omega_{R}^{2}-\left(p q \omega_{2}\right)^{2}}{\beta p q \omega_{2}}\right)=\arctan \left(\frac{\Omega_{R}^{2}-\left(p \omega_{1}\right)^{2}}{\beta p \omega_{1}}\right)=\chi_{p}, \\
\tilde{\delta}_{p}=-\arctan \left(\frac{p \omega_{1}}{\beta}\right)=\delta_{p q}, \\
\tilde{\psi}_{p}=\arctan \left(\frac{\beta}{p \omega_{1}}\right)=\psi_{p q} .
\end{gathered}
$$

Since Eqs. (28) and (29) agree with each other, we have shown that in the first-order approximation, the average velocity obtained by the $\mathrm{CC}$ theory is invariant if we interchange the additive and parametric forces, $q=\omega_{1} / \omega_{2}$ being a rational number different from $2 m /(2 n+1)(n=0,1,2, \ldots$ and $m=1,2, \ldots)$. On the contrary, if $q=2 m /(2 n+1)$ and $\omega_{1}$ $=q \omega_{2}$, the symmetry analysis of the partial differential equation (PDE) shows that the average velocity should be zero. However, interchanging these frequencies the kink can move with a nonzero ratchet velocity as has been recently shown in [27].

\section{RESULTS OF SIMULATIONS}

It should be stressed that, although the results obtained in Sec. II by using a symmetry analysis for Eqs. (1)-(3) are exact, they only predict whether our general parametric and additive periodic forces could induce a ratchet motion, i.e., from this symmetry analysis we obtain only a necessary condition for the appearance of unidirectional motion of the kink. On the other hand, the results obtained in Secs. III and IV by means of collective coordinate calculations are only approximate. Therefore in order to check the validity of our analytical results we must carry out numerical simulations for Eqs. (1)-(3).

To fully specify our system we must define explicitly the functions $g(t)$ and $f(t)$. In particular, we define the following periodic functions (period $T=2 \pi / \omega$ ):
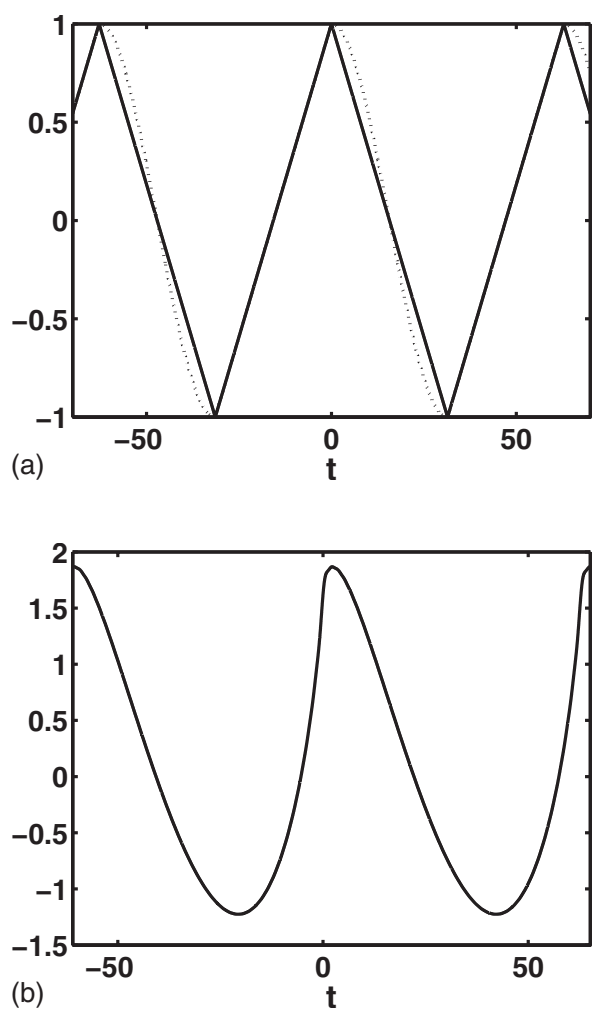

FIG. 1. Evolution of nonsinusoidal functions $h(t, \omega)$ (dotted line in the top panel), $-2 z(t, \omega)$ (solid line in the top panel), and $y(t, \omega, m)$ (bottom panel). Parameters: $\omega=0.1$ and $m=2$.

$$
\begin{gathered}
h(t, \omega)=\left\{\begin{array}{ll}
\cos (\omega t), & 0 \leq t \leq \frac{\pi}{\omega} \\
\frac{2 \omega}{\pi} t-3, & \frac{\pi}{\omega}<t<\frac{2 \pi}{\omega}
\end{array},\right. \\
z(t, \omega)=\left\{\begin{array}{ll}
\frac{\omega}{\pi} t-\frac{1}{2}, & 0 \leq t \leq \frac{\pi}{\omega} \\
-\frac{\omega}{\pi} t+\frac{3}{2}, & \frac{\pi}{\omega}<t<\frac{2 \pi}{\omega}
\end{array},\right. \\
y(t, \omega, m)=\sum_{k=1}^{40} \frac{1}{k^{m}}[\sin (k \omega t)+\cos (k \omega t)],
\end{gathered}
$$

where $h(t, \omega)$ and $z(t, \omega)$ are extended periodically in time, $h(t+T / 2, \omega) \neq-h(t, \omega), \quad y(t+T / 2, \omega, m) \neq-y(t, \omega, m) \quad$ (Fig. $1)$. Contrary to that, $z(t, \omega)$ is defined in such a way that it fulfills $z(t+T / 2, \omega)=-z(t, \omega)$ (top panel of Fig. 1). From this set of three functions we choose independently the nonsinusoidal driving forces $g(t)$ and $f(t)$.

In our first numerical test we check that, if $f\left(t+T_{2} / 2\right)$ $=-f(t)$, a net motion of the kink could appear only when the condition (9), implying $\omega_{1} / \omega_{2}=2 m /(2 n+1)$ with $m$ $=1,2,3, \ldots$ and $n=0,1,2, \ldots$, is violated. To this end, we take $g(t)=z\left(t, \omega_{1}\right)$ and $f(t)=z\left(t, \omega_{2}\right)$, and only change the relationship between the frequencies such that $\omega_{1} / \omega_{2}=1 / n$ or $\omega_{1} / \omega_{2}=n$, being $n=1,2, \ldots, 5$, fixing $\omega_{2}=0.1$ in the top 

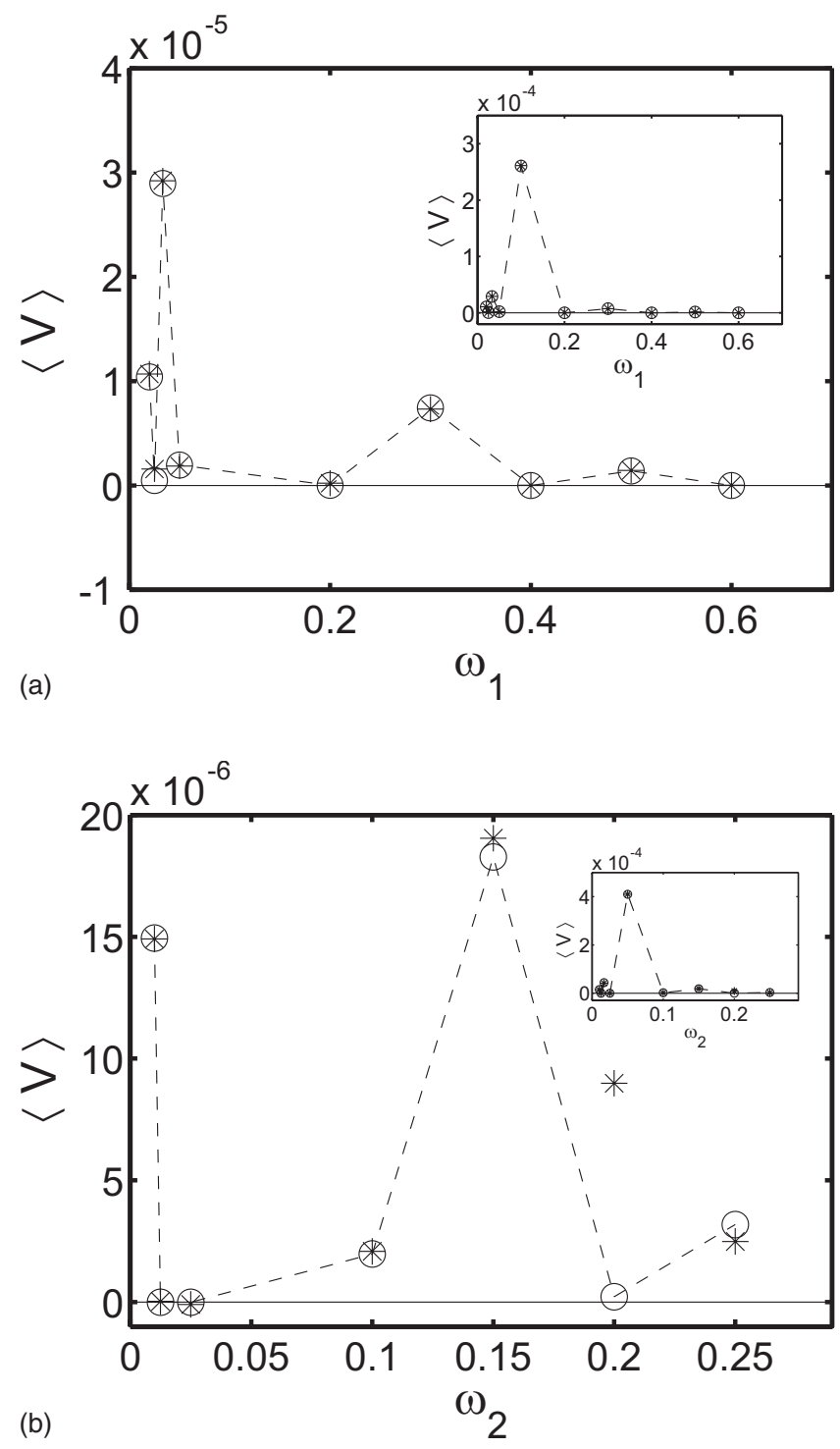

FIG. 2. Top panel: Average velocity versus $\omega_{1}\left(\omega_{1}=\omega_{2} / n\right.$ and $\left.\omega_{1}=n \omega_{2}\right)$ when $\omega_{2}=0.1$ and $n=1,2,3, \ldots 5$. The condition for zero average velocity (9) is fulfilled for $\omega_{1}=2 \omega_{2}, 4 \omega_{2}$, i.e., $\omega_{1}=0.2,0.4$. Bottom panel: Average velocity versus $\omega_{2}\left(\omega_{2}=n \omega_{1}\right.$ and $\left.\omega_{2}=\omega_{1} / n\right)$ when $\omega_{1}=0.05$ and $n=1,2,3, \ldots, 5$. For this choice of parameters Eq. (9) holds only for $\omega_{2}=\omega_{1} / 4, \omega_{1} / 2$, i.e., for $\omega_{2}=0.0125,0.025$ $\langle V\rangle=0$ is expected. Other parameters of the numerical simulations: $g(t)=z\left(t, \omega_{1}\right), f(t)=z\left(t, \omega_{2}\right), \epsilon_{1}=\epsilon_{2}=0.04$, and $\beta=0.1$. Open circles: numerical simulations of the PDE and stars: numerical solutions of the $\mathrm{CC}$ equations. In both cases the inset shows the results for all values of $n$, where the maximum average velocity is observed at $\omega_{1}=\omega_{2}$, while the figure magnifies the region of small velocities. The dashed lines connecting the circles and stars serve as guides for the eye.

panel of Fig. 2 and $\omega_{1}=0.05$ in the bottom panel of Fig. 2, respectively, and keeping the remaining parameters constant. Note that in the top panel the condition (9) for zero average velocity holds for $\omega_{1}=2 \omega_{2}, 4 \omega_{2}$, i.e., $\omega_{1}=0.2,0.4$, whereas in the bottom panel the average velocity is almost zero when $\omega_{2}=\omega_{1} / 4, \omega_{1} / 2$, i.e., for $\omega_{2}=0.0125,0.025$, as is expected.

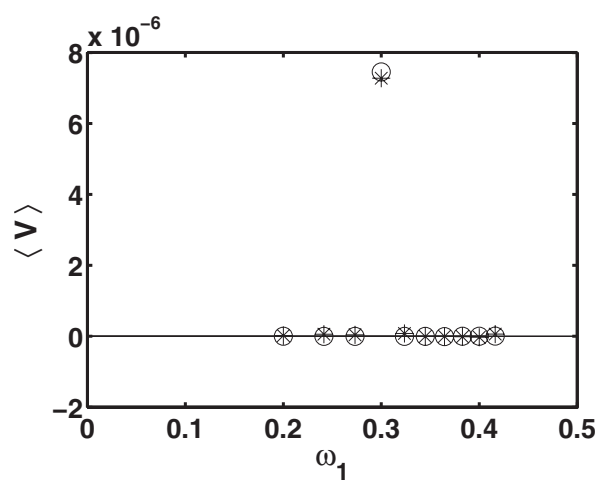

FIG. 3. Average velocity obtained by the numerical simulations of Eq. (1) versus the frequency of the additive periodic force. Open circles: numerical simulations of the PDE and stars: numerical solutions of the $\mathrm{CC}$ equations. Parameters of the numerical simulations are $\omega_{2}=0.1, \omega_{1}=\omega_{2}(1+\sqrt{n})$, with $n=1,2,3, \ldots, 10, \epsilon_{1}=\epsilon_{2}$ $=0.04$, and $\beta=0.1 . g(t)=z\left(t, \omega_{1}\right)$ and $f(t)=z\left(t, \omega_{2}\right)$ (see the text). As is expected a nonzero velocity is observed only when $n=4$.

In the following numerical test of expression (9) we change the relationship between the frequencies such that $\omega_{1} / \omega_{2}=1+\sqrt{n}$, with $n=1,2,3, \ldots, 10$ with $\omega_{2}=0.1$, keeping constant the rest of the parameters. In Fig. 3 we observe that, if $g(t)=z\left(t, \omega_{1}\right)$ and $f(t)=z\left(t, \omega_{2}\right)$, a nonzero average velocity only appears when $n=4\left(\omega_{1} / \omega_{2}=3\right)$. As expected, this relationship between the frequencies fulfills neither the condition (9) nor the condition (21) for zero average velocity. By contrast, for $n=1$ and $4\left(\omega_{1} / \omega_{2}=2\right.$ and $\left.\omega_{1} / \omega_{2}=4\right)$ and also for the values of $n$ such that $1+\sqrt{n}$ is an irrational number, the relations (9) or (21) hold and then net motion of the kink is not possible. This is also shown in Fig. 3 .

In the previous section we have also shown that, if $f(t)$ does not fulfill Eq. (8), the relationships between the frequencies obtained from (9) and (21) to assure zero average velocity are not valid, and therefore an average velocity different from zero can be expected when $\omega_{1}=\omega_{2}(1+\sqrt{n})$ or $\omega_{1}=n \omega_{2}$ for all values of $n$. Indeed, in contrast to the previous cases presented in Fig. 2 (top panel) and in Fig. 3, taking $g(t)=h\left(t, \omega_{1}\right)$ and $f(t)=h\left(t, \omega_{2}\right)\langle V\rangle \neq 0$ is observed in Fig. 4 for all the frequencies considered. It is interesting to compare the top panel of Fig. 2 with the bottom panel of Fig. 4: although the shapes of the driving forces we have used in each figure are very similar, as is shown in the top panel of Fig. 1, their relevant symmetry properties are different, and therefore in the former figure zero-average velocity is observed for $\omega_{1}=0.2 ; 0.4$, whereas in the latter one for all the frequencies considered in the simulations a zero-average velocity is not observed.

Finally we check that the average velocity is practically the same when the additive and parametric forces are interchanged. To this end we take the additive and parametric forces as $y\left(t, \omega_{2}, 2\right)$ and $y\left(t, \omega_{1}, 3\right)$, respectively, and after that we interchange them. In both cases, the numerical simulations of Eq. (1), the numerical solutions of the $\mathrm{CC}$ equations (13) and (14), and the analytical expression for the average velocity (29) are approximately the same as is shown in Fig. 5, where in the top and the bottom panel we take $\omega_{1}=\omega_{2}$ and $\omega_{1}=2 \omega_{2}$, respectively. 

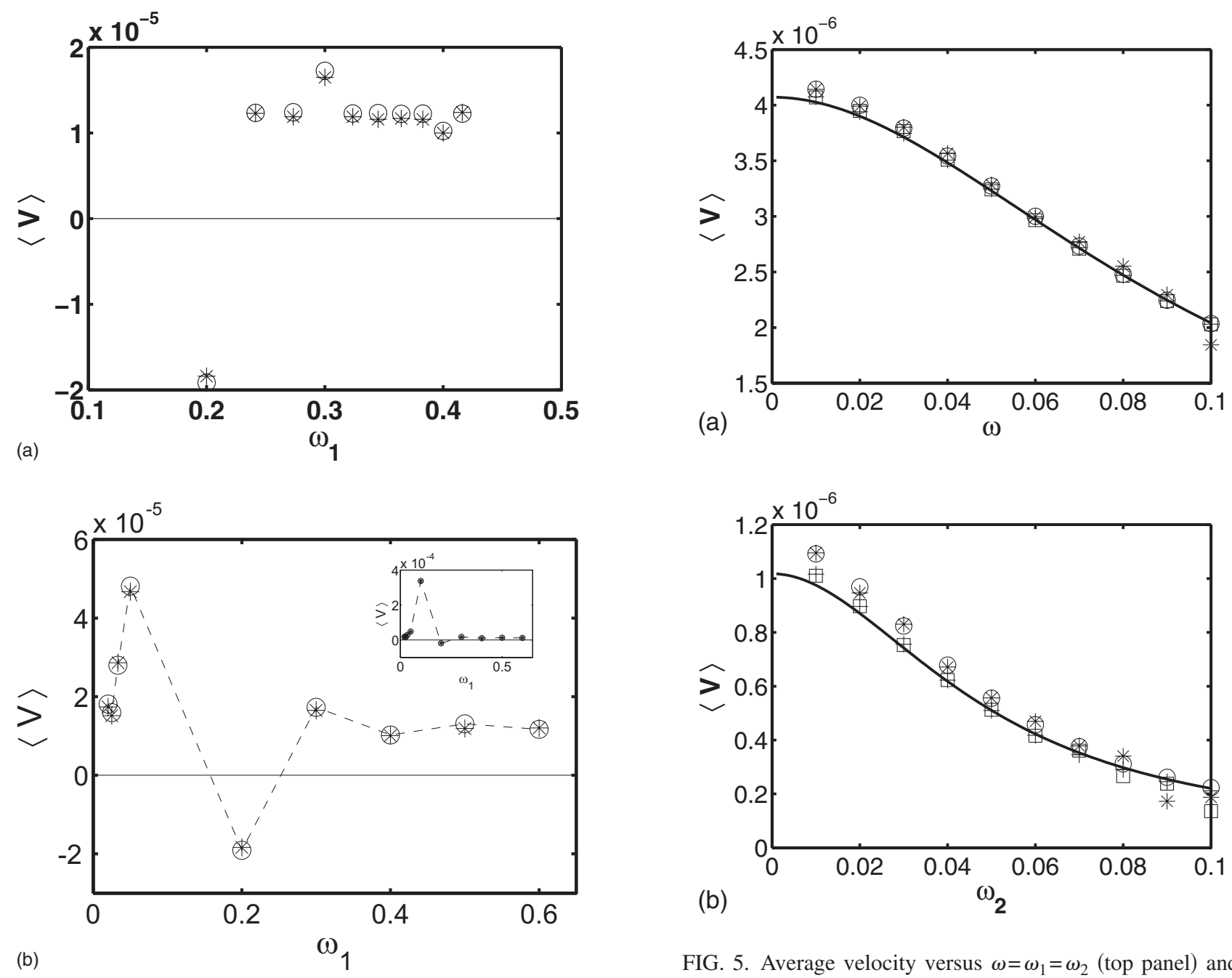

FIG. 4. Average velocity from the numerical simulations of PDE (1) versus frequency $\omega_{1}$ of the parametric periodic force, when the additive periodic force $f(t) \neq f\left(t+T_{2} / 2\right)$. Top panel: $\omega_{1}=\omega_{2}(1+\sqrt{n})$ with $n=1,2, \ldots, 10$, bottom panel: $\omega_{1}=n \omega_{2}$ and $\omega_{1}=\omega_{2} / n$ with $n$ $=1,2, \ldots, 5$. Open circles: numerical simulations of the PDE and stars: numerical solutions of the CC equations. Other parameters of the simulations: $g(t)=h\left(t, \omega_{1}\right), f(t)=h\left(t, \omega_{2}\right), \omega_{2}=0.1, \epsilon_{1}=\epsilon_{2}=0.02$, and $\beta=0.1$. Inset: results for all values of $n$, showing the maximum velocity when $\omega_{1}=\omega_{2}=0.1$, while the figure magnifies the region of small velocities. The dashed lines connecting the circles and stars serve as guides for the eye.

\section{CONCLUSIONS}

In this work we have studied the ratchetlike motion of the damped sine-Gordon kink under general periodic, additive, and parametric driving forces. Inspired by the symmetry analysis provided in $[18,28]$ we consider the symmetry transformations that leave our system invariant, but change the sign of the velocity. This approach allows us to find the relationship (9) between the frequencies of the parametric and additive driving forces, $\omega_{1}$ and $\omega_{2}$, respectively, in the case $f(t)=-f\left(t+T_{2} / 2\right)$ which is a necessary condition that must be broken in order to get a net motion of the kink. Indeed, we obtain that if $\omega_{1} / \omega_{2}=2 m /(2 n+1)$, being $m$

FIG. 5. Average velocity versus $\omega=\omega_{1}=\omega_{2}$ (top panel) and $\omega_{1}$ $=2 \omega_{2}$ (bottom panel). Open circles and stars: numerical simulations of Eq. (1) and numerical solutions of CC equations, respectively, when $f(t)=y(t, \omega, 2)$ and $g(t)=y(t, \omega, 3)$. Squares and pluses: numerical simulations of Eq. (1) and numerical solutions of CC equations, respectively, when $f(t)=y(t, \omega, 3)$ and $g(t)=y(t, \omega, 2)$. Solid lines represent $\langle V\rangle$ given by Eq. (29). Parameters: $\epsilon_{1}=\epsilon_{2}=0.001$ and $\beta=0.1$.

$=1,2,3, \ldots$ and $n=0,1,2, \ldots$ the average velocity of the kink must be zero. Using the Rice ansatz with two degrees of freedom, the center $X(t)$ and the width $l(t)$ of the kink, and using the Lagrangian formalism developed in [34], we obtain the equations of motion that satisfy $X(t)$ and $l(t)$. These equations of motion show that, although in the original system the average of the additive force is zero, the center of the kink "feels" an effective force with a nonzero average [see Eqs. (12) and (13)] [37,39] due to the oscillations of the kink width. This is also the ratchet mechanism that appears in other soliton ratchets in homogeneous sine-Gordon or $\phi^{4}$ equations [20,27,37]. Furthermore we have checked that the collective coordinate equations also preserve the symmetry properties that are relevant for the appearance of the ratchet effect. So the relationship between the frequencies of the parametric and additive driving forces given by Eq. (9) for the suppression of any net motion can also be obtained within the framework of the collective coordinates theory. In 
view of this result, by using the expression of the velocity obtained from the collective coordinate analysis we have found another relationship (21) between these two frequencies for zero-average velocity. We would like to stress that this second condition is not exact since it has been obtained by $\mathrm{CC}$ theory, however, the results of the numerical simulations of the PDE (1) and the numerical solutions of the CC equations (12) and (13) confirm its validity. These two conditions for the suppression of the net motion hold both for sinusoidal forces studied in Refs. [19,27,29] and for nonsinusoidal driving forces, which we have considered here. In both cases, it is interesting to note that the maximum average velocity appears when the frequencies of the parametric and additive driving forces coincide. This behavior is also observed in the case of nonsinusoidal forces either when they fulfill the same symmetry condition as the sinusoidal force, $f(t)=-f\left(t+T_{2} / 2\right)$ (see Figs. 2 and 3), or break it (Fig. 4).

Within the framework of the $\mathrm{CC}$ approach we have shown that when the first-order approximation holds, the average velocity is invariant if we interchange the additive $f(t)$ and parametric $g(t)$ forces, when $\omega_{1}=q \omega_{2}, q$ being a rational number different from $2 m /(2 n+1) \quad(n=0,1,2, \ldots$ and $m$ $=1,2, \ldots)$, i.e., $f(t) \neq-f\left(t+T_{2} / 2\right)$.

Another interesting conclusion is that the frequencies of the drivers can be used as a control parameter to change the direction of the ratchet motion. This characteristic not only appears in the ratchet systems we have considered here, but was also observed in other systems. Indeed, nonlinear signal mixing of two driving forces was used to control transport in overdamped particle ratchets [25], and current reversals due to the interplay between frequencies, phases, and amplitudes of two driving forces were obtained for sine-Gordon soliton ratchets with strongly localized inhomogeneities [26]. Such systems could now be investigated experimentally, because such inhomogeneities were already used in experiments on long Josephson junctions, namely, microresistors (critical current $J_{c}$ decreased) and microshorts $\left(J_{c}\right.$ increased) [40]. For the interpretation of such experiments our type of symmetry analysis will be very useful. The relationship between symmetries and transport has recently been investigated in experiments on ratchets for cold atoms [24], where a multifrequency driving has been used, obtained by combining signals at three different frequencies: $\omega_{1}, 2 \omega_{1}$, and $\omega_{2}$.

\section{ACKNOWLEDGMENTS}

We acknowledge financial support by the Ministerio de Educación y Ciencia (MEC, Spain) and by DAAD (Germany) through "Acciones Integradas Hispano-Alemanas" HA2004-0034-D/04/39957, and partially by MEC Grant No. FIS2005-973 (E.Z.S.,N.R.Q.), and by the Junta de Andalucía under Projects No. 00481, No. P06-FQM-01735, and No. FQM-0207 (E.Z.S.,N.R.Q.). E.Z.S. was supported by the University of Seville.
[1] J. Maddox, Nature (London) 369, 181 (1994); M. O. Magnasco, Phys. Rev. Lett. 71, 1477 (1993); see also Ratchets and Brownian Motors: Basics, Experiments and Applications, edited by H. Linke, special issue of Appl. Phys. A 75 (2002).

[2] I. Zapata, R. Bartussek, F. Sols, and P. Hänggi, Phys. Rev. Lett. 77, 2292 (1996).

[3] G. Carapella and G. Costabile, Phys. Rev. Lett. 87, 077002 (2001).

[4] F. Falo, P. J. Martínez, J. J. Mazo, and S. Cilla, Europhys. Lett. 45, 700 (1999); E. Trías, J. J. Mazo, F. Falo, and T. P. Orlando, Phys. Rev. E 61, 2257 (2000).

[5] A. V. Ustinov, C. Coqui, A. Kemp, Y. Zolotaryuk, and M. Salerno, Phys. Rev. Lett. 93, 087001 (2004).

[6] E. Goldobin, A. Sterck, and D. Koelle, Phys. Rev. E 63, 031111 (2001); for the experiments see M. Beck, E. Goldobin, M. Neuhaus, M. Siegel, R. Kleiner, and D. Koelle, Phys. Rev. Lett. 95, 090603 (2005).

[7] J. E. Villegas, S. Savelev, F. Nori, E. M. González, J. V. Anguita, R. García, and J. L. Vicent, Science 302, 1188 (2003).

[8] M. Schiavoni, L. Sánchez-Palencia, F. Renzoni, and G. Grynberg, Phys. Rev. Lett. 90, 094101 (2003); R. Gommers, S. Bergamini, and F. Renzoni, ibid. 95, 073003 (2005).

[9] P. Hänggi, F. Marchesoni, and F. Nori, Ann. Phys. 14, 51 (2005).

[10] P. Reimann and P. Häggi, Appl. Phys. A 75, 169 (2002).

[11] P. Hänggi and R. Bartussek, Nonlinear Physics of Complex System-Current Status and Future Trends, edited by J. Parisi et al., Lecture Notes in Physics Vol. 746 (Springer, Berlin, 1996), p. 476.

[12] R. D. Astumian and P. Hänggi, Phys. Today 55(11), 33 (2002).

[13] P. Reimann, Phys. Rep. 361, 57 (2002).

[14] Z. Farkas, P. Tegzes, A. Vukics, and T. Vicsek, Phys. Rev. E 60, 7022 (1999).

[15] L. Morales-Molina, F. G. Mertens, and A. Sánchez, Eur. Phys. J. B 37, 79 (2003); Phys. Rev. E 72, 016612 (2005).

[16] A. Ajdari, D. Mukamel, L. Peliti, and J. Prost, J. Phys. I 4, 1551 (1994).

[17] S. Flach, O. Yevtushenko, and Y. Zolotaryuk, Phys. Rev. Lett. 84, 2358 (2000).

[18] M. Salerno and Yaroslav Zolotaryuk, Phys. Rev. E 65, 056603 (2002).

[19] G. Costantini, F. Marchesoni, and M. Borromeo, Phys. Rev. E 65, 051103 (2002).

[20] L. Morales-Molina, N. R. Quintero, F. G. Mertens, and A. Sánchez, Phys. Rev. Lett. 91, 234102 (2003).

[21] A. Sánchez, L. Morales-Molina, F. G. Mertens, N. R. Quintero, J. Buceta, and K. Lindenberg, Fluct. Noise Lett. 4, L571 (2004).

[22] Mario Salerno and Niurka R. Quintero, Phys. Rev. E 65 025602(R) (2002).

[23] R. Gommers, P. Douglas, S. Bergamini, M. Goonasekera, P. H. Jones, and F. Renzoni, Phys. Rev. Lett. 94, 143001 (2005).

[24] R. Gommers, S. Denisov, and F. Renzoni, Phys. Rev. Lett. 96, 240604 (2006).

[25] S. Savel'ev, F. Marchesoni, P. Hänggi, and F. Nori, Europhys. Lett. 67, 179 (2004); Phys. Rev. E 70, 066109 (2004); M. 
Borromeo, P. Hänggi, and F. Marchesoni, J. Phys.: Condens. Matter 17, S3709 (2005).

[26] L. Morales-Molina, F. G. Mertens, and A. Sánchez, Phys. Rev. E 73, 046605 (2006).

[27] E. Zamora-Sillero, N. R. Quintero, and F. G. Mertens, Phys. Rev. E 74, 046607 (2006).

[28] S. Flach, Y. Zolotaryuk, A. E. Miroshnichenko, and M. V. Fistul, Phys. Rev. Lett. 88, 184101 (2002).

[29] A. L. Sukstanskii and K. I. Primak, Phys. Rev. Lett. 75, 3029 (1995); Yu. S. Kivshar and A. Sánchez, ibid. 77, 582 (1996).

[30] Ri. Chacón and N. R. Quintero, BioSystems 88, 308 (2007); see also F. G. Mertens, L. Morales-Molina, A. R. Bishop, A. Sánchez, and P. Müller, Phys. Rev. E 74, 066602 (2006).

[31] Bernardo Sánchez-Rey et al. (private communication).

[32] M. J. Rice and E. J. Mele, Solid State Commun. 35, 487 (1980).
[33] A. Sánchez and A. R. Bishop, SIAM Rev. 40, 579 (1998).

[34] N. R. Quintero and E. Zamora-Sillero, Physica D 197, 63 (2004); see also S. Cuenda and A. Sánchez, Chaos 15, 023502 (2005); J. Francisco Cao, E. Zamora-Sillero, and N. R. Quintero, Phys. Rev. E 73, 056603 (2006).

[35] N. R. Quintero, A. Sánchez, and F. G. Mertens, Phys. Rev. E 62, R60 (2000).

[36] N. R. Quintero and P. G. Kevrekidis, Phys. Rev. E 64, 056608 (2001).

[37] L. Morales-Molina, N. R. Quintero, A. Sánchez, and F. G. Mertens, Chaos 16, 013117 (2006).

[38] N. R. Quintero, A. Sánchez, and F. G. Mertens, Phys. Rev. E 64, 046601 (2001).

[39] N. R. Quintero, B. Sánchez-Rey, and M. Salerno, Phys. Rev. E 72, 016610 (2005).

[40] A. V. Ustinov, Phys. Lett. A 136, 155 (1989). 